\title{
Steady Marangoni Instability in a Liquid Layer with Deformable Free Surface in the Presence of Insoluble Surfactant and Internal Heat Generation Subject to Uniform Temperature
}

\author{
Razihan Allias, Mohd. Agos Salim Nasir and Seripah Awang Kechil
}

Faculty of Computer and Mathematical Sciences, UiTM Shah Alam, Malaysia

\begin{abstract}
The influence of internal heat generation on the onset of the Marangoni convection in a liquid layer system with insoluble surfactant is investigated. The liquid layer is bounded below by a uniformly heated plate and above by a deformable free surface. The surface tension is assumed to depend linearly on the surfactant concentration and the temperature of the liquid. The linear stability theory is used and the closed form analytical solution for the steady convection is obtained. The marginal curves are plotted to observe the effects of the physical parameters such as the elasticity number, internal heat source, Crispation number and Bond number on the onset of steady Marangoni instability. Surfactants and gravity waves stabilize the fluid system, but the internal heat generation and the surface deformability destabilize the system.
\end{abstract}

Keywords: Marangoni instability, Surfactant, Internal heat generation, Deformable surface, Uniform temperature, Analytical solutions.

\section{INTRODUCTION}

Thermocapillary convection and solutocapillary convection, known as Marangoni convection, are important in many areas of applications such as in industrial processes and fundamental sciences. The convective instability has been extensively studied starting with the pioneering experimental and theoretical works of Bénard (1900), Rayleigh (1916) and Pearson (1958). Pearson (1958) introduced the linear stability analysis to investigate theoretically the Marangoni instability in a fluid layer. Extensive review on the thermocapillary instability can be found in Davis (1987).

Marangoni instability can be affected by the existence of surface-active agent or also called as surfactant which can alter the surface tension of the fluid layer and act as a stabilizing factor. Mikishev and Nepomnyashchy (2010) studied the effect of insoluble surfactants on the long-wave Marangoni convection in a liquid layer for both steady and oscillatory convection in the case of nondeformable and deformable surface by using the linear stability theory and asymptotic expansion of the amplitudes of perturbation functions in terms of scaling parameter. It was found that the surface-active agent has stabilizing effect on the monotonic instability. Ab. Hamid et. al., (2011) considered the work of Mikishevand Nepomnyashchy (2010) to find the exact analytical solutions for the cases of thermally conducting and insulating bottom boundary and found that surfactant has greater stabilizing effect for the conducting case than insulating case.

Unlike surfactant which can act as a stabilizer, internal heat generation is one of the factors that are known to destabilize the liquid layer system. Char and Chiang (1994) studied the effect of internal heat generation on stability of Bernard-Marangoni convection. The internal heat generation acts as destabilizer. Char and Chiang (1997) investigated the couple effect of Coriolis force (rotating

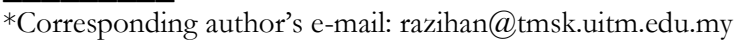


fluid) and internal heat generation on the onset of oscillatory instability of Bernard-Marangoni convection. Rotation stabilizes the fluid system while internal heat source has a destabilizing effect.

Nanjundappa et. al., (2011) considered the effect of internal heat generation on BernardMarangoniferroconvection instability in the presence of a uniform vertical magnetic field. The increasing magnetic Rayleigh number and internal heat generation have a destabilizing effect on the onset of BernardMarangoniferroconvection. The magnetic number has no effect on the onset of pure Marangoniferroconvection in the absence of internal heat generation. Hashim et. al., (2009) and Bachok and Ariffin (2011) used proportional linear thermal control feedback to delay the onset Marangoni convection and the controller reduces the destabilizing effect of the internal heat generation. Awang Kechil and Ab. Hamid (2012) considered the effect of insoluble surfactant and internal heat generation on the onset of Marangoni instability in a liquid layer with deformable free surface subject to a uniform heat flux. Stabilizing effect of the surfactant on the Marangoni instability was shown while internal heat generation destabilizes the liquid layer system.

Bhadhauria and Kiran (2014) theoretically study the combined effect of rotation speed modulation and internal heat generation in a temperature-dependent viscous fluid layer by employing nonlinear stability analysis and using Ginzburg-Landau model in a rotational speed modulation. It is found that internal heating destabilizes fluid layer system. The influence of internal heat generation has been analysed in Rayleigh-Benard convection in water well-dispersed with nanoliquids using linear and weakly nonlinear stability analysis (Kanchana and Zhao, 2018). The effect of internal heat generation is shown to promote or to supress the onset of convection. McCord et. al., (2016) generate the analytical solutions to the Stefan problem (solid-liquid phase change) with internal heat generation and compare the results with computational fluid dynamics analysis. The internal heat generation is used to separate the transient steady-state portions of the heat equation. The influence of the internal heat generation is studied by Yekasi et al. (2017) in a micropolar fluid with a Rayleigh-Benard convection together with the effect of temperature modulation. The Rayleigh-Benard convection is controlled by the effects of both heat generation and temperature modulation, either by advancing or delaying the convection.

In this paper, the influences of insoluble surfactant and internal heat generation on the onset of Marangoni instability in a horizontal liquid layer with deformable free surface subject to the uniform temperature are considered. The stability analysis theory is used, and the analytical solutions will be determined to assess the effects of the physical parameters on the critical Marangoni number. Marginal curves for steady convection will be analysed for the effects of elasticity number, internal heat generation, Crispation number and Bond number.

\section{PROBLEM FORMULATION}

Consider a horizontal liquid layer of thickness $d$ bounded below by a rigid plate at $z=0$ and the upper surface, $z=d$, is a deformable free surface. The bottom boundary has a noslip condition, and the temperature condition is set to have a fixed uniform temperature. The surface tension $\sigma$ is assumed to depend linearly on temperature and surfactant concentration.

$$
\sigma=\sigma_{\mathrm{o}}-\sigma_{1}\left(T-T_{\mathrm{o}}\right)-\sigma_{2}\left(\Gamma-\Gamma_{\mathrm{o}}\right)
$$

where $T$ and $\Gamma$ are the temperature of the liquid and the surfactant concentration, respectively. $\sigma_{0}$ is the reference surface tension corresponding to the reference temperature $T_{\mathrm{O}}$ and reference concentration $\Gamma_{\mathrm{o}}$ and $\sigma_{1}$ and $\sigma_{2}$ are positive constants.

For a very thin fluid layer or under microgravity conditions, the buoyancy effect can be neglected. Other physical properties of the liquid such as density, pressure, viscosity etc. are assumed to be constants. When the fluid is at rest, the hydrodynamic pressure with the atmospheric pressure $p_{a}$ and the gravitational acceleration $g$ at the reference steady state is

$$
p_{b}=p_{a}-\rho g(d-z)
$$

In the presence of internal heat source, the basic state temperature is in the form of parabolic distribution (Char and Chiang, 1994)

$$
T_{b}(z)=-\frac{q}{2 \chi} z^{2}+\left(\frac{q d}{2 \chi}-\frac{\Delta T}{d}\right) z+T_{\mathrm{o}},
$$

where $q$ is the uniformly distributed internal heat generation, $\Delta T$ is the temperature difference in the fluid 
across the layer and $\chi$ is the thermal diffusivity. If there is no internal heat generation $(q=0)$, the basic state temperature distribution is linear (Mikishev and Nepomnyashchy, 2010). The governing equations of the liquid system are

$$
\begin{gathered}
\nabla \cdot \mathbf{v}=\mathbf{0} \\
\frac{\partial T}{\partial t}+(\mathbf{v} \cdot \nabla) T=\chi \nabla^{2} T+q \\
\frac{\partial \mathbf{v}}{\partial t}+(\mathbf{v} \cdot \nabla) \mathbf{v}=-\frac{\nabla p}{\rho}-v \nabla^{2} \mathbf{v}+\boldsymbol{g}
\end{gathered}
$$

which represent the equations of continuity (mass), energy and momentum, respectively.

$\mathbf{v}=(u, v, w)$ is the fluid velocities in the $(x, y, z)$ directions, $T$ is the temperature, $\boldsymbol{g}$ is the gravitational field, $v$ is the kinematic viscosity, $p$ is the pressure, $\rho$ is the density and $t$ is the time. The surfactant distribution at the free surface (Mikishev and Nepomnyashchy, 2010), is

$$
\frac{\partial \Gamma}{\partial t}+\nabla_{s} \cdot\left(u_{\tau} \Gamma\right)+K u_{n} \Gamma=D_{\mathrm{o}} \nabla_{s}^{2} \Gamma,(7)
$$

where $u_{n}$ and $u_{\tau}$ are normal and tangential velocities, respectively, $D_{\mathrm{O}}$ is the surfactant diffusivity, $K$ is the local surface curvature and $\nabla_{s}$ is the surface gradient. In the linear stability analysis, the introduction of infinitesimal perturbation, linearization, scaling and superposition of the normal modes transform the partial differential equations into system of ordinary differential equations. The nondimensional linearized momentum and heat equations are,

$$
\frac{1}{\operatorname{Pr}} r\left(D^{2} W-k^{2} W\right)=D^{4} W-2 k^{2} D^{2} W+k^{4} W
$$

$$
D^{2} \theta-k^{2} \theta-r \theta=[1-Q(1-2 z)] W
$$

The no-slip and temperature conditions at the bottom surface $Z=0$,

$$
\begin{gathered}
W=D W=0, \\
\theta=\mathbf{o} \text { (uniform temperature). }
\end{gathered}
$$

At the upper free surface, $Z=1$,

$$
W+r Z=0
$$

$$
D \theta+\operatorname{Bi} \theta=\operatorname{Bi}(Q+1) Z,
$$

$$
\begin{aligned}
& \frac{1}{P r} r D W+3 k^{2} D W-D^{3} W+\frac{k^{2}}{C r}\left(B o+k^{2}\right) Z=0, \text {, 14) } \\
& \left(D^{2}+k^{2}\right) W+M k^{2} \theta-M k^{2} Z(Q+1) \\
& \quad+N k^{2} \frac{N D W}{r+k^{2} L}=0
\end{aligned}
$$

Where $W$ and $\theta$ are the velocity and temperature amplitudes, respectively, and $k$ is the wave number. The nondimensional parameters are $B i=h d / \lambda$, $L=D_{\mathrm{o}} / \chi, B o=g \rho d^{2} / \sigma_{1}, M=\sigma_{1} d \Delta T / \chi \mu$, $\operatorname{Pr}=v / \chi, C r=\rho \chi v / \sigma_{\mathrm{O}} d, \quad N=\sigma_{2} d^{2} \Gamma_{\mathrm{o}} / \chi \mu$, $Q=q d^{2} / 2 \chi \Delta T$, which represent the Biot number, Lewis number, Bond number, Marangoni number, Prandtl number, Crispation number, elasticity number, and internal heat generation, respectively. $Z$ is the surface deflection and $\mathrm{D}=d / d z$.

The onset of steady Marangoni convection can be determined by solving the system of equations (8) - (15). The analytical solutions for the steady Marangoni convection will be determined and the marginal curves will be plotted to assess the effects of the parameters on the critical Marangoni number.

\section{ANALYTICAL SOLUTIONS}

The exact analytical solutions for the steady Marangoni convection of the linearized problem (8) - (15) subject to conducting lower boundary layer are obtained with the aid of Maple algebra package by setting $r=0$ where the stability is at marginal state. The critical Marangoni number is obtained to determine the onset of Marangoni instability.

The solutions $W(z)$ and $\theta(z)$ for the system are

$$
\begin{gathered}
W(z)=C_{1}[-k z \cosh (k z)+\sinh (k z)(1-z) \\
+\sinh (k z) z k \operatorname{coth} k],
\end{gathered}
$$




$$
\begin{aligned}
& \theta(z) \\
& =\frac{C_{3} \exp (k z)+C_{2} \exp (-k z)-\left(C_{1} / 6\right) \exp (-k z)}{k^{4} \sinh k} \\
& \{[(A+B+C) \exp (2 k z)-A+B+C] \\
& \cosh [k(2 z-1)]+[(-A-B+C+3 Q / 8) \\
& \exp (2 k z)-A+B+C-3 Q / 8] \sinh [k(2 z-1)] \\
& +[(D+E+3 Q / 8) \exp (2 k z)+\mathrm{D}-\mathrm{E}+3 \mathrm{Q} / 8] \\
& \cosh [\mathrm{k}(1+2 \mathrm{z})]+[(-D-E) \exp (2 k z) \\
& +D+E+3 Q / 8] \sinh [\mathrm{k}(1+2 \mathrm{z})] \\
& +[(-F z k+G) \sinh k+(F k z \cosh k)] z k^{3} \exp (2 k z) \\
& +\left[F z^{2} k^{4}+G z k^{3}+[(9 Q / 8)-9 / 8] k^{2}\right. \\
& +[(15 Q / 8)-3 / 8] k+3 Q / 4] \sinh k \\
& +k \cosh k\left[(3 / 4+(z-3 / 4) Q) z^{2} k^{3}\right. \\
& \left.\left.+z^{2} k^{3}+(-3 Q / 8+3 / 8) k-3 Q / 4\right]\right\},
\end{aligned}
$$

where

$$
\begin{aligned}
A & =\frac{3 z k^{3}}{2}\left[\frac{1}{2}+\left(z-\frac{1}{2}\right) Q\right], \\
B & =\left(-\frac{3}{4}+\frac{21}{8} z-\frac{3}{4} z^{2}\right) Q-\frac{3 z}{8}+\frac{3}{4}, \\
C & =\left[-\frac{3}{16}+\left(\frac{21}{16}-\frac{3}{4} z\right) Q\right] k, \\
D & =\frac{3 k^{2}(z-1)}{4}\left[\frac{1}{2}+\left(z-\frac{1}{2}\right) Q\right] k^{2}, \\
E & =\left[\frac{3}{16}+\left(\frac{3}{4} z-\frac{9}{16}\right) Q\right] k, \quad F=\frac{3}{4}+\left(z-\frac{3}{4}\right) Q, \\
G & =\left(\frac{9}{4} z+\frac{3}{2}-z^{2}\right) Q-\frac{3}{4} z+\frac{3}{2}
\end{aligned}
$$

$$
\begin{aligned}
C_{3}= & \frac{1}{48 k^{4} \sinh k(k \cosh k+B i \sinh k)} \\
& \left\{C _ { 1 } \left[18 Q k^{2}-6 k^{2}+\cosh ^{2} k\left(6 k^{2}+3 k^{3}\right.\right.\right. \\
& -2 k^{4} Q-12 Q k^{2}-3 Q k^{3}+6 k^{4}-3 B i k^{2} \\
& \left.+6 B i Q-3 B i k+15 B i Q k+3 B i Q k^{2}\right) \\
& +\cosh k \sinh k\left(-3 k^{2}-3 k^{3}-6 Q k+3 Q k^{3}\right. \\
& +15 Q k^{2}+3 B i k^{2}+6 B i k^{3}-18 B i Q k \\
& \left.-2 B i Q k^{3}-3 B i Q k^{2}\right) \\
& +\sinh k\left[24 B i Z k^{4}(1+Q)\right] \\
& +C_{1}\left(8 Q k^{4}+3 B i k^{2}-6 B i Q+3 B i k\right. \\
& \left.\left.\left.+6 B i k^{4}+9 B i Q k^{2}-15 B i Q k+2 B i Q k^{4}\right)\right]\right\}, \\
C_{2}= & \frac{1}{16 k^{4} \sinh k}\left\{C_{1} \cosh k\left(3-3 Q k^{2}-2 Q k\right)\right. \\
& -\sinh k\left[C_{1}\left(3 Q k^{2}-15 Q k-3 k^{2}-3 k+2 Q\right)\right. \\
& \left.\left.-16 C_{3} k^{4}\right]\right\},
\end{aligned}
$$

and $C_{1}$ is an arbitrary constant. The closed form of analytical solution for the Marangoni number $M$ is

$$
\begin{aligned}
M & =\frac{-12\left(B o+k^{2}\right) k}{L H}\left[\left(2 L k^{2} \cosh ^{2} k-B i N\right.\right. \\
& \left.+B i N \cosh ^{2} k-2 k^{2} B i L+k^{2} B i N\right) \sinh k(18) \\
& -\left(2 k B i L+2 k^{3} L+k^{3} N+k N\right) \cosh k \\
& \left.+(k N+2 k L B i) \cosh ^{3} k\right],
\end{aligned}
$$

where

$$
\begin{aligned}
H & =\left(-4 k^{3} Q B o-4 k^{5} Q-9 k^{3} Q\right. \\
& \left.+3 k B o-9 k Q B o+3 k^{3}\right) \sinh k \\
& +\left(-3 k^{2} Q B o-3 k^{3} Q k-3 k^{3} Q k\right. \\
& -3 k B o) \cosh h^{2} k \sinh k \\
& -\left(24 k^{6} C r-Q k^{4} B o-6 Q k^{2} B o\right. \\
& +6 Q B o-Q k^{6}-6 k^{2} Q \cosh ^{2} k \\
& +24 k^{6} C r Q-3 k^{6}-3 k^{4} B o \\
& \left.-6 \cosh ^{2} k Q B o+6 k^{2} Q-6 Q k^{4}\right) \cosh k .
\end{aligned}
$$




\section{RESULT AND DISCUSSIONS}

The marginal curves for the steady convective instabilities are in the plane $(k, M)$ which separate the region of stability and the region of instability. The onset of convection sets is determined by the global minimum of $M$ denoted by $M_{c}$.

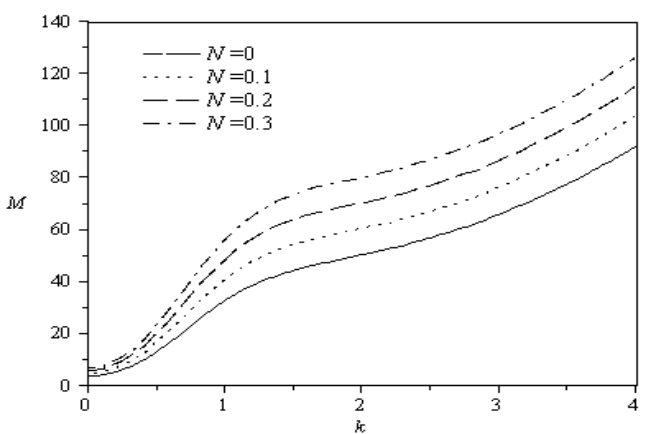

Figure 1. Stability curves for steady convection with $Q=1$, $C r=0.01$, and $L=B i=B o=0.1$, for various values of elasticity number.

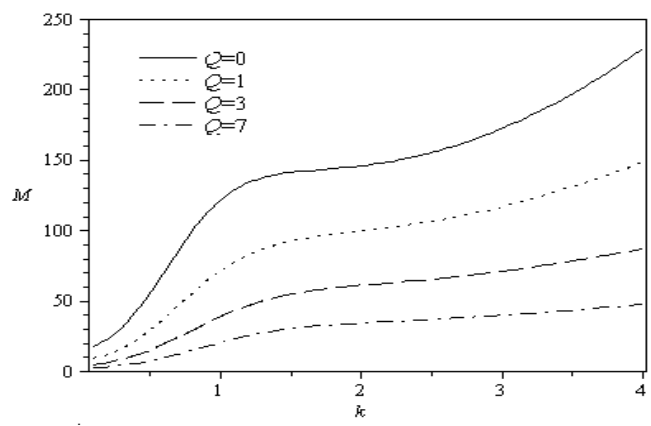

Figure 2. Stability curves for steady convection with $C r=0.01, N=0.5$ and $L=B i=B O=0.1$ for various values of internal heat source.

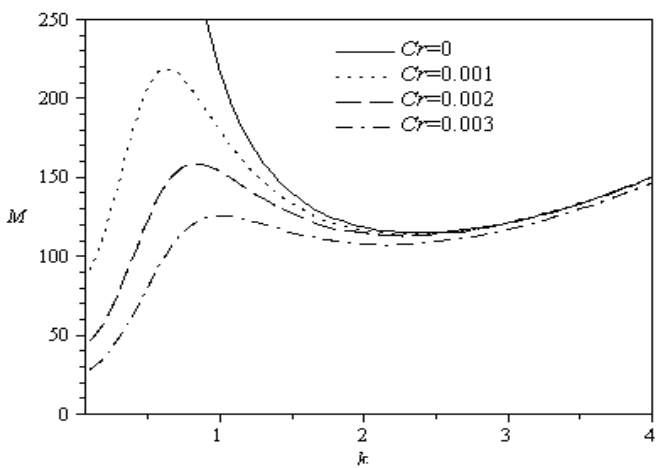

Figure 3. Stability curves for steady convection with $Q=1$, $N=0.5$ and $L=B i=B O=0.1$ for various values of Crispation number.

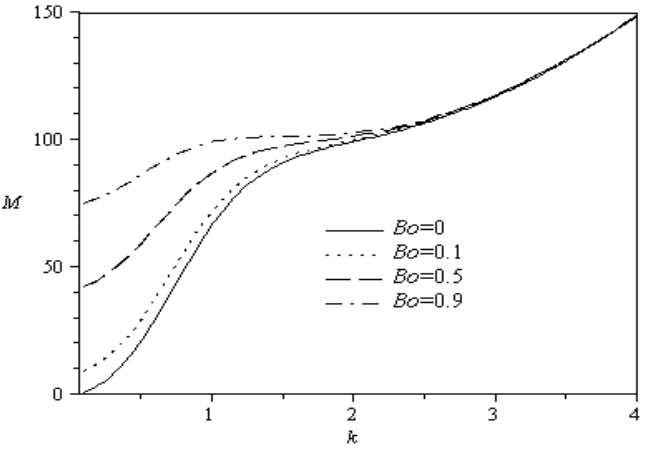

Figure 4. Stability curves for steady convection with $C r=0.01, Q=1, N=0.5$ and $L=B i=0.1$ for various values of Bond number.

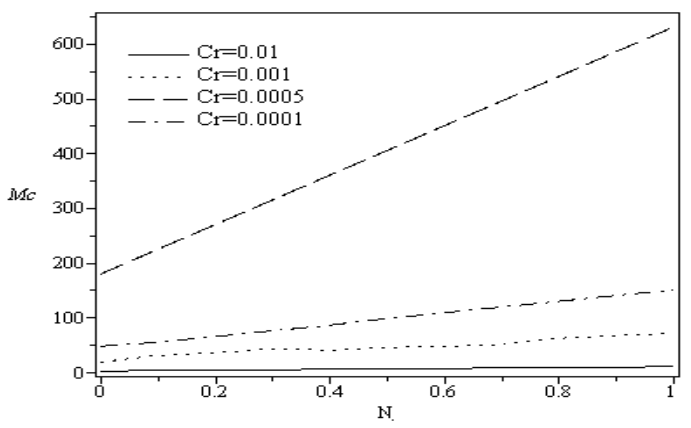

Figure 5. The effect of elasticity number on the critical Marangoni number for various values of Crispation number with $Q=1$ and $L=B o=B i=0.1$.

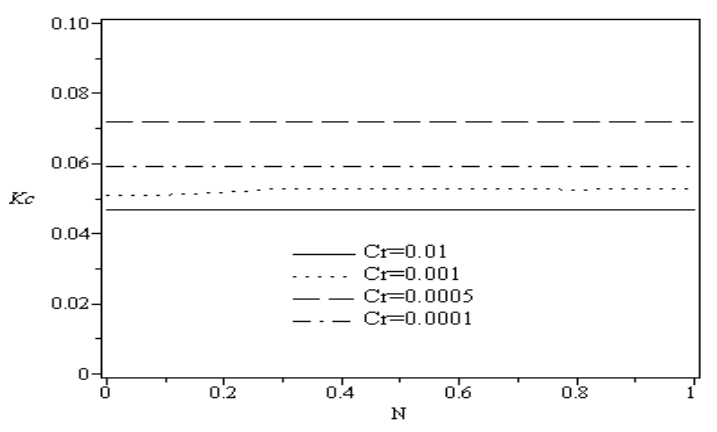

Figure 6. The effect of elasticity number on the critical wave number for various values of Crispation number with $Q=1$ and $L=B O=B i=0.1$. 


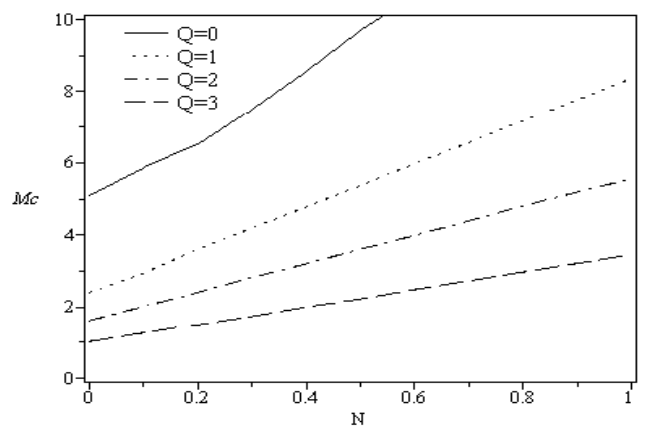

Figure 7. The effect of elasticity number on the critical Marangoni number for various values of internal heat generation with $C r=0.01$ and $L=B o=B i=0.1$.

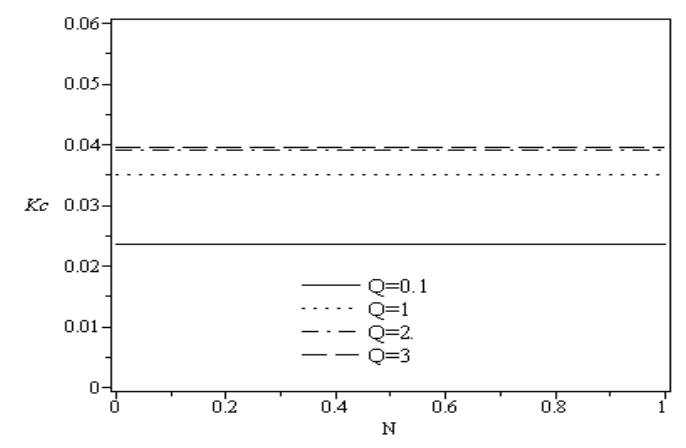

Figure 8. The effect of elasticity number on the critical wave number for various values of internal heat generation with $C r=0.01$ and $L=B o=B i=0.1$.

Figure 1 shows the effect of the elasticity parameter $N$ on the steady curves with the parameters' values $Q=1$, $C r=0.01$ and $L=B i=B o=0.1$. In the absence of surfactant, $N=0$, the curve has the lowest critical value of the Marangoni number, $M_{c}$. As the value of the elasticity number increases, the value of Marangoni number $M_{c}$ also increases, and hence, the surfactants stabilize the fluid layer system. An opposite effect occurs when the liquid layer generates internal heat, $Q$ as shown in Figure 2. Internal heat generation has a destabilizing effect on Marangoni instability as the increased value of the internal heat source decreases the value of the critical Marangoni number $M_{c}$. Without internal heat generation, $Q=0$, the fluid layer is at its highest stability.

The rigidity of the deformable surface of the fluid layer system is determined by the value of Crispation number, $\mathrm{Cr}$. Figure 3 shows the marginal stability curves for the effect of $\mathrm{Cr}$ on the critical Marangoni number $M_{c}$ where increasing value of $\mathrm{Cr}$ will decrease the value of $\mathrm{M}_{c}$. If $\mathrm{Cr}=$ o (flat surface), the liquid layer is at its highest stability.
For a deformable surface, $M_{c}$ is at the long wavelength $(k=0)$ and reduces as the Crispation number increases. The system of liquid layer is more stable if the degree of the deformability is low.

As shown in Figure 4, the increasing values of Bond number will increase the value of the critical Marangoni number $M_{c}$. The Bond number measures the effect of gravity waves at the free surface and the result shows that Bond number has a significant stabilizing effect. Figure 5 and Figure 6 show the effect of the elasticity number on the critical Marangoni number $M_{c}$ and the critical wave number $k_{c}$, respectively, for various values of Crispation number. The values of the critical Marangoni number is almost linearly increasing as the values of elasticity number increasing but the Crispation number has a destabilizing effect on the layer system. Surface deformability destabilizes the fluid layer system. However, the elasticity number has little influence on the value of the critical wave number $k_{c}$. High surface deformability reduces the wave number as shown in Figure 6 . The reduction of the value of the internal heat source will increase the value of the critical Marangoni number $M_{c}$. The value of the critical Marangoni number increases proportionally with the values of elasticity number as shown in Figure 7. In Figure 8, the effect of increasing heat source reduces, and the elasticity number has negligible effect on the critical wave number $k_{c}$

\section{CONCLUSION}

The steady Marangoni instability is examined by obtaining the exact analytical solutions of the system of equations for a liquid layer with deformable free surface subject to uniform temperature in the presence of insoluble surfactant and internal heat generation. The surface tension is assumed to depend linearly on the temperature and the surfactant concentration of the fluid layer system. It is found that the surfactant and gravity waves (Bond number) have stabilizing effects. Internal heat source and surface deformability act as destabilizing factors. The fluid system is most stable when there is no internal heat generation, and the upper surface is flat. 


\section{ACKNOWLEDGEMENT}

The authors would like to express sincere gratitude to the Faculty of Computer and Mathematical Sciences, University Teknologi MARA (600IRMI/DANA5/3/LESTARI(0140/2016)) for the fund and support.

\section{REFERENCES}

Ab. Hamid, A. S., Awang Kechil, A. S., \&Abd. Aziz, A. S. 2011, Marangoni Instability in a fluid layer with insoluble surfactant. World Academy of Science, Engineering and Technology, 58, 24-28.

Awang Kechil, S.\& Ab. Hamid, A. S. 2012, Steady Marangoni instability in a fluid layer with insoluble surfactant and internal heat generation. Advances in Mathematical and Computational Methods, 125-135.

Bénard, H. 1900, Les tourbillons cellulaires dans une nappe liquid. Revue Générale des Sciences Pures et Appliquées,11,1261-1271.

Bachok, N. \& Ariffin, N. M. 2011, Feedback control of the Marangoni-Bernard convection in a horizontal fluid layer with internal heat generation. Microgravity Science and Technology, 22, 97-105.

Bhadauria, B. S. \&Kiran, P. 2014, Effect of rotational speed modulation on heat transport in a fluid layer with temperature dependent viscosity and internal heat source. Ain Shams Engineering Journal, 54, 35-42.

Char, M. I. \& Chiang, K. T. 1994, Stability analysis of Bernard-Marangoni convection in fluids with internal heat generation. J. Phys. D: Appl., 27, 748-755.

Char, M. I. \&Chiang, K. T. 1997, Oscillatory instability analysis of Bernard-Marangoni convection in rotating fluid with internal heat generation. Int. J. Heat Mass Transfer, $40,857-867$.

Davis, S.H. 1987, Thermocapillary instabilities. Annual Review of Fluid Mechanic, 19, 403-405.

Hashim, I., Othman, H. \& Awang Kechil, S. 2009, Stabilization of thermocapillary instability in a fluid layer with internal heat source. International Communications in Heat and Mass Transfer, 36, 161-165.
Kanchana, C. \& Zhao, Y. 2018, Effect of internal heat generation/absorption on Rayleigh-Bernard convection in water well-disperse with nanoparticles or carbon nanotubes. International Journal of Heat and Mass Transfer,

McCord, D., Crepeau, J., Siahpush, A., \& Brogin, J. A. F. 2016, Analytical solutions to the Stefan problem with internal heat generation. Applied Thermal Engineering, $103,443-451$.

Mikishev, A. B. \& Nepomnyashchy, A. A. 2010, Longwavelength Marangoni convection in a liquid layer with insoluble surfactant: lineartheory. Microgravity Science and Technology, 22, 415-423.

Nanjundappa, C. E., Shivakumara, I. S., \& Arukumar, R. 2011, Onset of Bernard-Marangoniferroconvection with internal heat generation. Microgravity Science and Technology, 23, 29-39.

Pearson, J. R. A. 1958, On convection cells induced by surface tension. Journal of Fluid Mechanics, 4, 489-500.

Rayleigh, L. 1916, On convection currents in a horizontal layer of fluid with the higher temperature is on the other side. Philosophical Magazine. 32, 529-543.

Yekasi. V., Pranesh, S., \& Bathul S. 2017, Effect of temperature modulation and internal heat generation on the onset of Rayleigh-Bernard convection in a micropolar fluid. Global Journal of Pure and Applied Mathematics, 13, 2411-2437. 